\title{
Short- and Long-Term Stability of Lyophilised Melatonin-Loaded Lecithin/Chitosan Nanoparticles
}

\author{
Anita Hafner, ${ }^{*, a}$ Marjana Dürrigl, ${ }^{b}$ Ivan Pepić, ${ }^{a}$ and Jelena Filipović-GrČIĆ ${ }^{a}$ \\ ${ }^{a}$ Department of Pharmaceutics, Faculty of Pharmacy \& Biochemistry, University of Zagreb; 10000 Zagreb, Croatia: and \\ ${ }^{b}$ Research and Development, PLIVA Croatia Ltd.; 10000 Zagreb, Croatia. \\ Received April 20, 2011; accepted June 6, 2011; published online June 9, 2011
}

\begin{abstract}
The aim of this study was to establish a freeze-drying process for melatonin-loaded lecithin/chitosan nanoparticles (NPs) to preserve their chemical and physical stability for a longer time period that what is possible in an aqueous suspension. Glucose and trehalose were investigated as potential excipients during freeze-drying of NP suspensions. Lecithin/chitosan NPs were characterised by mean diameter and zeta potential, ranging between 117.4 and $328.5 \mathrm{~nm}$ and 6.7 and $30.2 \mathrm{mV}$, respectively, depending on the lecithin type and chitosan content in the preparation. Melatonin loadings were up to $7.1 \%$. For all lecithin/chitosan NPs, no notable differences in the mean particle size, size distribution, zeta potential or melatonin content were observed before or immediately after the lyophilisation process or after 7 months of storage at $4{ }^{\circ} \mathrm{C}$. The residual moisture contents of lyophilisates with glucose and trehalose immediately after the lyophilisation process varied between $4.0-4.8 \%$ and 2.4-3.0\%, respectively. All lecithin/chitosan NPs had a fully amorphous nature after the freeze-drying process, as indicated by modulated differential scanning calorimetry. NP lyophilisates with glucose had a low glass transition temperature $\left(\mathrm{ca} .5^{\circ} \mathrm{C}\right)$, confirming that lyophilisation with glucose as a cryoprotectant was not appropriate. All lyophilisates with trehalose had a glass transition temperature above the room temperature, allowing formation of the cake without a collapse of the structure, which was capable of preserving its characteristics and appearance following 7 months of storage at $4{ }^{\circ} \mathrm{C}$.
\end{abstract}

Key words nanoparticle; freeze-drying; cryoprotectant; chitosan; lecithin; melatonin

Recently, attention has been paid to lecithin/chitosan nanoparticles (NPs) as an alternative colloidal carrier system to polymeric nanoparticles, solid lipid nanoparticles, liposomes and nanoemulsions. These nanoparticles were obtained from the supra-molecular self-organising interaction of the negatively charged lipid material lecithin and the positively charged polysaccharide chitosan, without preliminary vesicle formation. ${ }^{1,2)}$ These constitutive materials are favoured due to their well-known characteristics: Chitosan is a biocompatible and biodegradable cationic polysaccharide exhibiting bioadhesive and penetration enhancing properties ${ }^{3)}$; lecithin is a natural lipid mixture of phospholipids, mainly phosphatidylcholine and phosphatidylethanolamine, and is considered a safe and biocompatible excipient that has been frequently used for the preparation of various delivery nanosystems, such as micro- and nanoemulsions, ${ }^{4)}$ liposomes ${ }^{5)}$ micelles $^{6)}$ and solid lipid nanoparticles. ${ }^{7)}$

Mucoadhesive lecithin/chitosan NPs were shown to be suitable carriers for lipophilic drugs. NP suspension prepared with Lipoid S45 lecithin with a lecithin-to-chitosan weight ratio of 20:1 enhanced melatonin permeability through a Caco-2 cell monolayer compared to its permeability in an aqueous solution, without inducing plasma membrane damage or a decrease in cell viability at the concentration tested ${ }^{8}{ }^{8}$ In another study, lecithin/chitosan NP dispersion was shown to increase accumulation of clobetasol-17-propionate in the skin without any significant permeation across the skin, compared to chitosan gel and commercial cream. ${ }^{9}$ These findings imply that lecithin/chitosan NPs have great potential to improve the permeation of encapsulated drugs across various biological barriers. However, the overall poor stability of colloidal nanoparticles in an aqueous medium is a significant drawback from a development perspective. Thus, one of the most important challenges in the development of nanoparticles is to achieve long-term stability during storage.

Freeze-drying is a well-established approach to increase the chemical and physical stability of colloidal systems over extended time periods. Prior to the freeze-drying process, special excipients are usually added to the formulation to protect the nanoparticles from various stresses generated during the freezing and drying steps. The role of sugars, such as sorbitol, mannitol, trehalose, glucose, sucrose, and lactose, as lyoprotective and/or cryoprotective agents to prevent nanoparticle aggregation and improve the redispersibility of the dry product has been reviewed in the literature. ${ }^{10)}$ Briefly, stabilisation of materials in sugar glasses has been explained by the formation of a glassy sugar matrix, which acts as a physical barrier between the particles ${ }^{11)}$ and inhibits diffusion on a relevant time scale. ${ }^{12)}$ Recently, Zhang et al. reported that sugars such as glucose and trehalose are very effective in preventing particle aggregation and inhibiting leakage of an active ingredient during the solid lipid microparticle freezedrying process. ${ }^{13)}$ For cationic nanoparticles, interactions with lyoprotective agents were reported to influence nanoparticle biological activity. ${ }^{14)}$

Aqueous suspensions of melatonin-loaded lecithin/chitosan NPs were shown to be stable up to a two-month period at $4{ }^{\circ} \mathrm{C}{ }^{8)}$ However, it is not possible to preserve their chemical and physical stability for a longer time period in that form. Therefore, the aim of this study was to establish a freeze-drying process for lecithin/chitosan NPs to further improve their physico-chemical stability. Glucose and trehalose were investigated at concentrations of $1,2.5,5$ and $10 \%$ $(\mathrm{w} / \mathrm{v})$ as potential excipients and stabilisers during freezedrying of NP suspensions. The impact of the type and amount of the cryoprotectant, the type of lecithin and the lecithin-to-chitosan weight ratio in the NP suspensions on the stability and resuspension of lyophilised product was evalu- 
ated. Nanoparticles were characterised in terms of size, zeta potential and melatonin content before and after the lyophilisation process. Further characterisation of freeze-dried NPs included determining the residual moisture and thermal analysis.

\section{Experimental}

Reagents and Chemicals The following materials were used as received: chitosan in the form of hydrochloride salt Protasan $^{\circledR}$ UP CL 113, deacetylation degree $86 \%$; molecular weight (MW) $50000-150000 \mathrm{~g} / \mathrm{mol}$; Novamatrix, Norway); soybean lecithins (commercial mixtures of lipids, phospholipids, and fatty acids; a kind gift from Lipoid GmbH, Germany); Lipoid S45 (fat free soybean lecithin with $45 \%$ phosphatidyl choline); Lipoid S100 (phosphatidyl choline from fat free soybean lecithin) and melatonin (Sigma, U.S.A.). All other chemicals or solvents used were of analytical grade and purchased from Kemika (Croatia).

Preparation of Lecithin/Chitosan NPs Lecithin (Lipoid S45 or Lipoid $\mathrm{S} 100)$ was dissolved in $96 \%$ ethanol at a $2.5 \%(\mathrm{w} / \mathrm{v})$ concentration. Melatonin was dissolved in the ethanolic solution of lecithin at a $0.5 \%(\mathrm{w} / \mathrm{v}) \mathrm{con}-$ centration, obtaining a lecithin-to-melatonin weight ratio of $5: 1$. Chitosan was solubilised in distilled water at a $1 \%(\mathrm{w} / \mathrm{v})$ concentration. Melatoninloaded NP suspensions were obtained by injection of $4 \mathrm{ml}$ ethanolic lecithin/melatonin solution (syringe inner diameter of $0.75 \mathrm{~mm}$ ) into $46 \mathrm{ml}$ of water-diluted chitosan solutions and magnetically stirred at $900 \mathrm{rpm}$. Appropriate volumes of a $1 \%$ chitosan solution were diluted with distilled water prior to ethanolic lecithin/melatonin solution injection to obtain lecithin to chitosan weight ratios (L/C) of $20: 1$ and $10: 1$ in the prepared NP suspensions.

For comparison, melatonin-loaded Lipoid S45 and S100 lecithin NPs were prepared by injection of ethanolic melatonin/lecithin solutions into distilled water.

Determination of Melatonin Loading Encapsulation efficiency (EE \%) was measured by determining the amount of non-entrapped melatonin using the dialysis technique as previously reported ${ }^{8)}$ Briefly, an appropriate volume $(4 \mathrm{ml})$ of the melatonin-loaded NP suspension was placed into a dialysis bag of cellulose acetate (Spectra/Por ${ }^{\circledR}$ membranes, MW cut off $\left.12-14000\right)$ and dialysed against distilled water $(100 \mathrm{ml})$, magnetically stirred at $30 \mathrm{rpm}$. Experiments were performed under sink conditions (melatonin water solubility $0.1 \mathrm{mg} / \mathrm{ml}$ ). At scheduled time intervals, $2-\mathrm{ml}$ samples were withdrawn from the receiver solution and replaced with fresh water. Samples were diluted with acetonitrile to obtain a water-to-acetonitrile volume ratio of $45: 55$ and then analysed for melatonin content by HPLC. The experiment was stopped when constant drug concentration values were detected in subsequent withdrawals from the receiver phase, taking into account the progressive dilution of the medium.

Entrapment efficiency (EE \%) was then calculated according to the following equation:

\section{$\mathrm{EE} \%=([$ total drug $]-[$ diffused drug $]) /[$ total drug $] \times 100$}

Drug loading (DL \%) was calculated according to the following equation:

$$
\mathrm{DL} \%=([\text { total drug] }-[\text { diffused drug] }) / \text { examined quantity of NPs } \times 100
$$

The dialysed NP suspensions samples (without non-entrapped melatonin) were used in all further studies.

Physical Characterisation of the Particle Size and Surface Charge Size and zeta potential of NPs were measured by Photon Correlation Spectroscopy (PCS) using Zetasizer 3000 HS (Malvern Instruments, Malvern, U.K.) following dilution with $0.45 \mu \mathrm{m}$ filtered distilled water and $10 \mathrm{~mm}$ $\mathrm{NaCl}$ solution, respectively. The $\mathrm{pH}$ of diluted samples ranged from $5.8-$ 6.0. Zeta-potential measurements were done at $25^{\circ} \mathrm{C}$. Samples were placed in the electrophoretic cell, where a potential of $150 \mathrm{mV}$ was established.

Freeze-Drying Parameters The effect of the cryoprotective agents trehalose and glucose on the stability of melatonin-loaded NPs during freezedrying was determined. The NP concentration in the suspensions was kept constant $(2 \mathrm{mg} / \mathrm{ml})$. Trehalose or glucose was added to the NP suspensions at concentrations of $1,2.5,5$ and $10 \%(\mathrm{w} / \mathrm{v})$. NP suspensions without cryoprotective agents were also prepared as reference samples. After the preparation, aliquots of NP formulations were transferred to glass vials for freezedrying. The samples were frozen at $-70{ }^{\circ} \mathrm{C}$ for $3 \mathrm{~h}$. After freezing, NP dispersions were uncapped and transferred into a $-50^{\circ} \mathrm{C}$ preequilibrated chamber of an Alpha 1-4 freeze-dryer (Martin Christ, Germany), which was depressurised to $5 \mathrm{~Pa}$ using a vacuum pump (Vacuubrand RZ2, Germany). The temperature was kept at $-50^{\circ} \mathrm{C}$ during $24 \mathrm{~h}$ of primary drying. Secondary drying was conducted at $20^{\circ} \mathrm{C}$ for $8 \mathrm{~h}$. After reestablishment of ambient pressure, vials were capped, weighed and stored at $4{ }^{\circ} \mathrm{C}$ until further use.

Determination of Residual Moisture The residual moisture content of the lyophilised formulations was measured by Karl Fischer titration using a Mettler DL 38 titrator (Mettler-Toledo, Switzerland). Samples containing $20 \mathrm{mg}$ of each formulation in the presence of $2.5 \%(\mathrm{w} / \mathrm{v})$ glucose and trehalose were analysed directly after the freeze-drying process and after 7 months of storage at $4{ }^{\circ} \mathrm{C}$. The moisture content of the nanoparticle powders was monitored in triplicate.

Reconstitution of Lyophilised Samples The original volume of deionised distilled water filtered through a membrane filter $(0.2 \mu \mathrm{m})$ was added to each vial, which was then vortexed $(3 \times 30 \mathrm{~s})$ to rehydrate the freeze-dried sample.

After reconstitution, nanoparticles were characterised by the methods described below.

Characterisation of Melatonin-Loaded NPs after Reconstitution After reconstitution in distilled water, NP suspensions were analysed in terms of average diameter and zeta potential of the NPs. To determine melatonin retention, $1.5-\mathrm{ml}$ aliquots of reconstituted samples were dialysed against $30 \mathrm{ml}$ distilled water for $30 \mathrm{~min}$, and the concentration of free melatonin (representing leakage) was determined using HPLC. ${ }^{8)}$

Modulated Differential Scanning Calorimetry (MDSC) Modulated differential scanning calorimetry (MDSC) analyses were performed on a TA Instrument Q1000 Modulated DSC (TA Instruments, New Castle, DE, U.S.A.) using aluminium hermetic pans with pierced lids to allow for removal of residual solvent, with approximately $5 \pm 2 \mathrm{mg}$ sample, under a dynamic nitrogen atmosphere $(50 \mathrm{ml} / \mathrm{min})$. Samples were heated at $5^{\circ} \mathrm{C} / \mathrm{min}$ from -20 to $160^{\circ} \mathrm{C}$ using a modulation temperature amplitude of $\pm 0.8^{\circ} \mathrm{C}$ and period of $60 \mathrm{~s}$. The glass transition temperature $\left(T_{\mathrm{g}}\right)$ was determined using the TA Universal Analyses 2000 Software by extrapolating the linear portion of the DSC curve above and below the glass transition point and determining the midpoint temperature in the reverse heat flow curve. The analyses were performed in triplicate.

Stability Studies Lyophilised samples were stored in closed vials at $4{ }^{\circ} \mathrm{C}$ for a period of seven months. At scheduled time points, characterisation of stored freeze-dried NPs (residual moisture determination and thermal analysis) was performed. Lyophilisates were resuspended in the original volume of distilled water and NPs were characterised in terms of size, zeta potential and melatonin content.

HPLC Assay of Melatonin Quantification of melatonin was performed by HPLC. The system consisted of a SCL-10Avp system controller, LC10Advp pump, DGU-14A degasser, SPD-10AVvp UV-VIS detector, CTO10Avp column oven (Shimadzu, Japan) and Pathfinder ${ }^{\mathbb{B}} \mathrm{C} 18$ AP column $(4.6 \times 150 \mathrm{~mm}$; Shimadzu). The mobile phase consisted of acetonitrile/water $(55: 45, \mathrm{v} / \mathrm{v}){ }^{15)}$ The flow rate was $1 \mathrm{ml} / \mathrm{min}$, oven temperature $40^{\circ} \mathrm{C}$ and injection volume $20 \mu \mathrm{l}$. The column eluant was monitored at $229 \mathrm{~nm}$. Melatonin peaks were separated with a retention time of $2 \mathrm{~min}$.

X-Ray Analyses Powder X-ray diffraction data were recorded on a Philips X'Pert PRO diffractometer (PAN Analytical, Germany) equipped with a $X^{\prime}$ Celerator detector $\left(2.022^{\circ} 2 \theta\right)$ using $\mathrm{Cu} K \alpha$ radiation at $45 \mathrm{kV}$ and $40 \mathrm{mV}$. Scan angle range $(2 \theta)$ was $2-50^{\circ}$, step size $(2 \theta)$ was $0.017^{\circ}$ and time per step was $50 \mathrm{~s}$. Samples are applied directly onto a Phillips' original circular sample holder (16 mm diameter) and closed with the bottom plate. Diffractograms were analyzed using X'Pert Data Collector software.

Statistical Analysis Statistical data analyses were performed on all data by one-way analysis of variance (ANOVA) followed by multiparametric Tukey's post-hoc test with $p<0.05$ as the minimal level of significance. Calculations were performed using the GraphPad Prism program (GraphPad Software, Inc., San Diego, U.S.A.; www.graphpad.com).

\section{Results and Discussion}

Characterisation of NPs The composition and main characteristics of the NPs prepared are summarised in Table 1. Four samples of melatonin-loaded lecithin/chitosan NP suspensions were prepared by injection of ethanolic lecithin/melatonin solution into an aqueous chitosan hydrochloride solution, varying the type of lecithin (Lipoid S100 and Lipoid S45) and the lecithin-to-chitosan weight ratio employed $(20: 1$ and $10: 1)$. In all preparations, the melatonin-to-lecithin weight ratio was $5: 1$. 
Table 1. Influence of Lecithin Type (Lipoid S100 and S45) and Lecithin-to-Chitosan Ratio (L/C) on the Main Characteristics of Melatonin-Loaded Lecithin/Chitosan NPs

\begin{tabular}{|c|c|c|c|c|c|c|c|c|c|c|}
\hline \multirow{3}{*}{$\begin{array}{c}\mathrm{L} / \mathrm{C} \\
(\mathrm{w} / \mathrm{w})\end{array}$} & \multicolumn{10}{|c|}{ Type of lecithin } \\
\hline & \multicolumn{5}{|c|}{ Lipoid S100 } & \multicolumn{5}{|c|}{ Lipoid S45 } \\
\hline & NP & $\begin{array}{l}\text { Drug loading } \\
(\%)^{a)}\end{array}$ & Size (nm) & PDI & $\begin{array}{l}\text { Zeta-potential } \\
\qquad(\mathrm{mV})\end{array}$ & NP & $\begin{array}{l}\text { Drug loading } \\
(\%)^{a)}\end{array}$ & Size (nm) & PDI & $\begin{array}{l}\text { Zeta-potential } \\
\qquad(\mathrm{mV})\end{array}$ \\
\hline $1 / 0$ & A1 & $3.9 \pm 0.2$ & $87.2 \pm 0.8$ & 0.27 & $-11.0 \pm 2.1$ & $\mathrm{C} 1$ & $4.8 \pm 0.3$ & $90.5 \pm 1.5$ & 0.32 & $-35.6 \pm 1.3$ \\
\hline $20 / 1$ & $\mathrm{~A} 2$ & $4.8 \pm 0.1$ & $117.4 \pm 3.9$ & 0.39 & $6.7 \pm 1.6$ & $\mathrm{C} 2$ & $6.2 \pm 0.2$ & $241.2 \pm 3.3$ & 0.21 & $26.4 \pm 5.6$ \\
\hline $10 / 1$ & A3 & $4.8 \pm 0.2$ & $122.8 \pm 0.8$ & 0.47 & $14.7 \pm 1.1$ & $\mathrm{C} 3$ & $7.1 \pm 0.2$ & $328.5 \pm 3.1$ & 0.30 & $30.2 \pm 0.8$ \\
\hline
\end{tabular}

PDI=Polydispersity index. Values are mean \pm S.D. $(n=3) .{ }^{*}$ Does not differ from corresponding lecithin NPs $(p>0.05)$. The samples of NPs that were included in residual moisture and stability studies are shaded. $a$ ) Drug loading $\%=([$ total drug] $-[$ diffused drug] $) /$ examined quantity of NPs $\times 100$.

Between the two lecithins used, Lipoid S45 has a higher content of lipids that contribute to its negative charge, giving rise to its interaction with positively charged chitosan. The overall negative charge of Lipoid S45 lecithin and the consequent formation of chitosan/lecithin NPs through their electrostatic interaction have already been described in the literature. ${ }^{1,2)}$

NPs were separated from unentrapped melatonin using the dialysis method. The main NP characteristics, size and surface charge, were determined using dialysed systems diluted with water and $10 \mathrm{~mm} \mathrm{NaCl}$, respectively. $\mathrm{pH}$ values in diluted dialysed systems were between 5.8 and 6.0.

The main NP characteristics presented in Table 1 are consistent with those reported in our previous study. ${ }^{8)}$ Briefly, the lecithin/chitosan NPs obtained were characterised by mean diameters ranging between $117.4 \pm 3.9$ and $328.5 \pm 3.1 \mathrm{~nm}$ and were significantly larger than corresponding lecithin NPs. The zeta potential was inverted from negative values for lecithin NPs to positive values for lecithin/chitosan NPs, especially for NPs prepared with Lipoid S45 lecithin (up to $30.2 \mathrm{mV}$ ). The size and the surface charge of NPs increased with chitosan content and the negative charge of lecithin used in the preparation. Compared to lecithin NPs, lecithin/chitosan NPs had significantly higher melatonin loadings (up to $7.1 \%$, w/w; $p<0.05$ ).

In our previous study, it was shown that the aqueous suspensions of melatonin-loaded lecithin/chitosan NPs were stable for one to two months at $4{ }^{\circ} \mathrm{C}$. ${ }^{8}$ ) To further improve the physico-chemical stability of melatonin-loaded NPs, suspensions were subjected to a freeze-drying process.

Main Characteristics of Lecithin/Chitosan NPs after Freeze-Drying and Reconstitution Preliminary studies revealed that freeze-drying of lecithin/chitosan NP suspensions in the absence of any excipient resulted in particle aggregation and a drastic increase in the polydispersity index (data not shown). Thus, in the current study, different excipients (trehalose, glucose, sucrose and mannitol) were added at different concentrations $(1,2.5,5$ and $10 \%, w / v)$ to NP suspensions prior to freeze-drying to prevent particle aggregation and to improve the redispersibility of the dry product. The concentration of NPs in all of the suspensions that were prepared was $0.2 \%(\mathrm{w} / \mathrm{v})$. NPs were characterised in terms of their size and polydispersity before and after freeze-drying in the presence of each excipient at the concentrations tested. According to the preliminary results, trehalose and glucose were more effective in preserving NP stability during lyophilisation and subsequent reconstitution than were the other two excipients, sucrose and mannitol (data not shown). This finding is in agreement with literature reports indicating that trehalose has achieved special status among freeze-drying stabilising excipients for biomaterials.

The advantages of trehalose over other sugars include lower hygroscopicity, an absence of internal hydrogen bonds and consequently more flexible formation of hydrogen bonds with nanoparticles during freeze-drying, very low chemical reactivity and a higher glass transition temperature $T_{\mathrm{g}} \cdot{ }^{10,16,17)}$ The extensive use of glucose as a stabiliser to protect nanoparticles against freezing and the drying stresses has been reported in the literature. ${ }^{10,18,19)}$ Furthermore, it was shown that a minimal concentration of $2.5 \%(\mathrm{w} / \mathrm{v})$ of trehalose or glucose was needed for suitable reconstitution of nanoparticles lyophilised at the particle concentration of $0.2 \%(\mathrm{w} / \mathrm{v})$. Therefore, subsequent studies focused on the influence of the lecithin type and presence of chitosan on the stability and resuspension of lyophilised product using trehalose and glucose as cryoprotectants at the concentration of $2.5 \%(\mathrm{w} / \mathrm{v})$.

The main characteristics of NPs before and after freezedrying in the presence of trehalose or glucose $(2.5 \%, \mathrm{w} / \mathrm{v})$ and rehydration are shown in Fig. 1.

All formulations in the presence of a cryoprotective agent could easily be redispersed. As for all lecithin/chitosan NPs (A2, A3, C2 and C3 samples), no considerable difference in the mean particle size, size distribution, zeta potential or melatonin content before and after freeze-drying and reconstitution were observed. Similar observations were already reported in the literature: comparable mean particle diameters, polydispersities and zeta potentials before and after freeze-drying have been reported for positively charged chitosan/poly $(\gamma$-glutamic acid) nanoparticles lyophilised in the presence of 1.5 or $2.5 \%$ trehalose $^{20)}$ and for chitosan/TPP nanoparticles lyophilised in the presence of 2.5 or $5 \%$ glucose. ${ }^{19)}$ However, a deviation higher than $10 \%$ in the mean NP diameter before and after freeze-drying was observed for the chitosan-free lecithin NPs (samples A1 and C1; Fig. 1). Thus, the initial size of lecithin NPs prepared with Lipoid S100 (sample A1; 87.2 $\pm 0.8 \mathrm{~nm}$ ) decreased to $72.0 \pm 0.6 \mathrm{~nm}$ after freeze-drying in the presence of $2.5 \%$ glucose $(\mathrm{w} / \mathrm{v})$, whereas the initial size of lecithin NPs prepared with Lipoid S45 (sample C1; $90.5 \pm 1.5 \mathrm{~nm}$ ) increased to $151.3 \pm 5.9$ or $101.2 \pm 2.1 \mathrm{~nm}$ after freeze-drying in the presence of trehalose or glucose $(2.5 \%$; $/ v)$, respectively. These results suggest that lecithin/chitosan NPs are more resistant to the freeze-drying process than are lecithin NPs. 
(a)

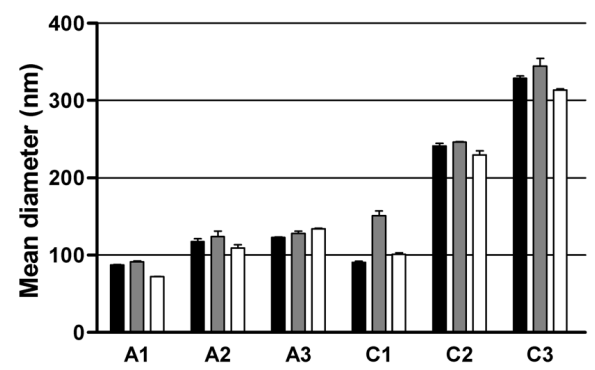

(c)

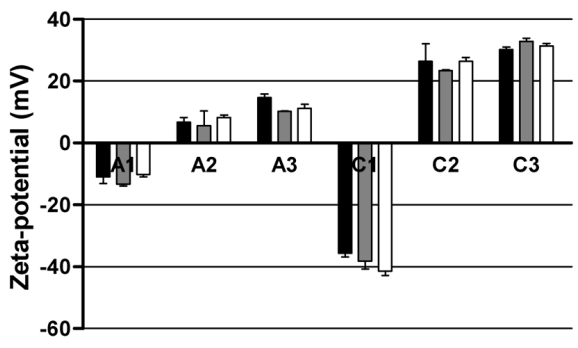

(b)

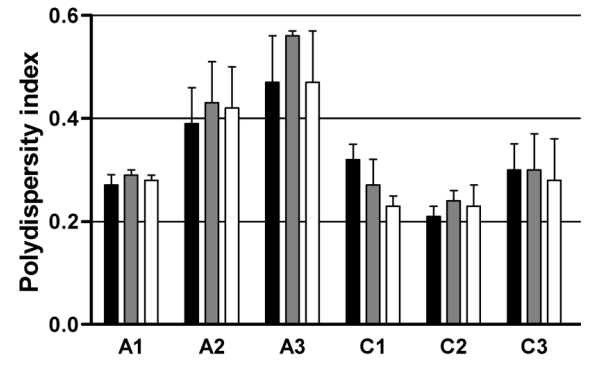

(d)

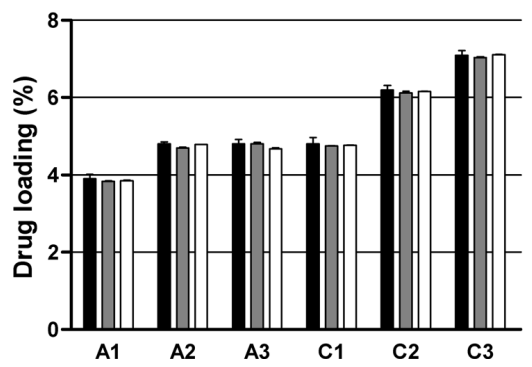

Fig. 1. Mean Diameter (a), Polydispersity (b), Zeta Potential (c) and Drug Loading (d) of Melatonin-Loaded NPs before ( $\square$ ) and Immediately after FreezeDrying in the Presence of Trehalose $(2.5 \%(\mathrm{w} / \mathrm{v}), \square)$ or Glucose $(2.5 \%(\mathrm{w} / \mathrm{v}), \square)$ and Rehydration in the Original Volume

Data are expressed as mean \pm S.D. $(n=3)$.
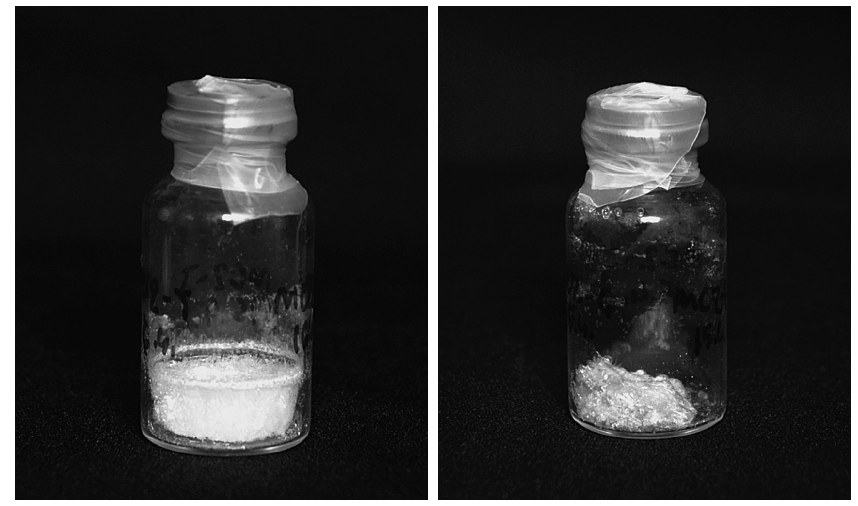

Fig. 2. Representative Freeze-Dried Products: Lipoid S45 Lecithin/Chitosan NPs with a Lecithin-to-Chitosan Weight Ratio of 20:1 (Sample C2) Lyophilised in the Presence of Trehalose $(2.5 \%(\mathrm{w} / \mathrm{v})$, Left) and Glucose $(2.5 \%(\mathrm{w} / \mathrm{v})$, Right)

A critical analysis of a freeze-dried product also includes the final volume and the cake appearance. ${ }^{21)}$ For lecithin/chitosan NPs lyophilised in the presence of trehalose or glucose, the structure of the dry product depended on the nature of the excipient used. When trehalose was used as cryoprotectant, an intact cake occupying the same shape and volume as the original frozen suspension was obtained. In contrast, the final freeze-dried cake containing glucose as cryoprotectant was partially collapsed (Fig. 2). As previously mentioned, the size of lecithin/chitosan NPs remained unchanged after freezedrying in the presence of glucose, despite the collapse of the cryoprotectant matrix. Although no NP aggregation occurred, such collapse can impact the product quality, prolong the time of reconstitution and increase the residual moisture content. $^{22)}$

Residual Moisture Content The residual moisture content of the freeze-dried formulation is a good primary quality
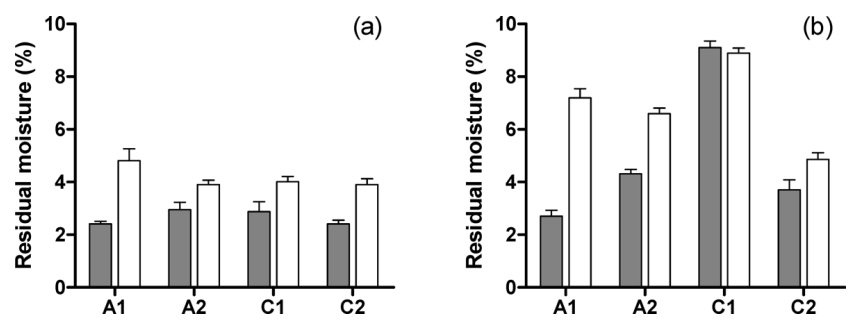

Fig. 3. Residual Moisture Content of Freeze-Dried NP Formulations Containing Trehalose $(2.5 \%(\mathrm{w} / \mathrm{v}), \square)$ and Glucose $(2.5 \%(\mathrm{w} / \mathrm{v}), \square)$ Immediately after Drying (a) and after 7 Months of Storage at $4{ }^{\circ} \mathrm{C}$ (b)

indicator and plays a critical role in long-term physical and chemical stability. A significant change in moisture content upon storage may be due to various reasons, such as stopper moisture release, amorphous excipient crystallisation and moisture release from excipient hydrate. ${ }^{23)}$ Thus, the residual moisture content of the freeze-dried formulations was measured immediately after the lyophilisation process and after 7 months of storage at $4{ }^{\circ} \mathrm{C}$. Immediately after the lyophilisation process, all formulations were characterised by the residual moisture content below 5\% (Fig. 3a). NPs lyophilised in the presence of trehalose had lower residual moisture contents $(2.4-3.0 \%)$ than did NPs lyophilised in the presence of glucose $(4.0-4.8 \%)$. This could be due to the fact that intact lyophilisation cakes obtained when trehalose was used as cryoprotectant ensured sufficient water removal. At the same time, higher residual moisture content of NPs lyophilised in the presence of glucose was a consequence of the partial collapse of the formulations. ${ }^{22,24)}$

Thermal Properties Solid-state properties of lyophilised formulations are assessed using MDSC. All lecithin and lecithin/chitosan NPs displayed a fully amorphous nature after freeze-drying. Reverse heat flow curves obtained for the lyophilised lecithin NPs with trehalose dis- 

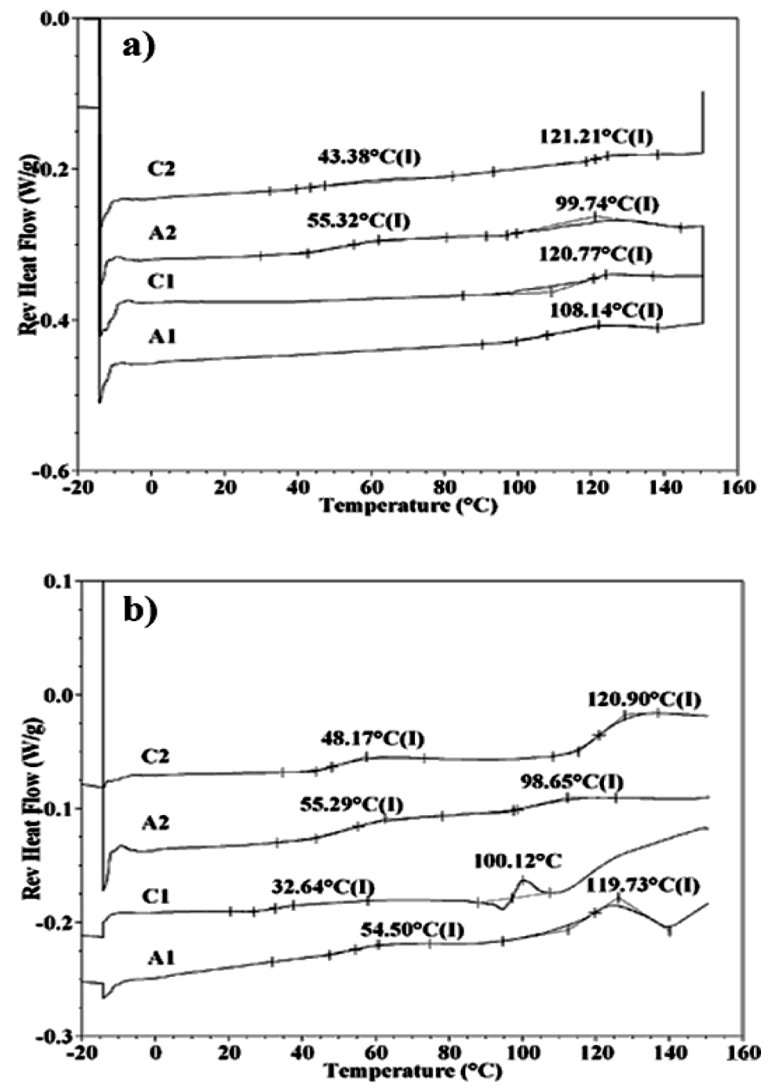

Fig. 4. Reverse Heat Flow Curves Obtained for the Freeze-Dried NP Formulations with Trehalose Immediately after Drying (a) and after 7 Months of Storage at $4{ }^{\circ} \mathrm{C}$ (b)

played a broad thermal event appearing in the region of $108-120^{\circ} \mathrm{C}$ (Fig. 4a). This thermal event was attributed to the glass transition of amorphous trehalose that corresponded well to already reported values. ${ }^{12,16,24-26)}$ These data also suggest that the mixing tendency of lecithin NPs and trehalose is high, as a single amorphous phase was formed upon lyophilisation. However, such mixing could have induced perturbation of the structure of lecithin NPs, causing deviation of nanoparticle size after reconstitution. The reverse heat flow curves obtained for the lyophilised lecithin/chitosan NPs with trehalose revealed two thermal events, first in the region of $43-55^{\circ} \mathrm{C}$ and the second in region of $99-121^{\circ} \mathrm{C}$ (Fig. 4a). By analysing corresponding physical mixtures, the first event was attributed to the NPs themselves, whereas the second thermal event originated from the trehalose. These results suggest that mixing of lecithin/chitosan NPs with trehalose was not fully accomplished and that two distinct phases were present in the lyophilisate. However, such NPs were unchanged after reconstitution, preserving the main NP characteristics unaffected. In addition, whether fully or partially miscible, all formulations had a glass transition temperature above room temperature and allowed appropriate cake formation without collapsed structure.

Thermal properties of lechitin/chitosan NP lyophilisates with glucose displayed extremely low glass transition temperatures, beginning at $c a .5^{\circ} \mathrm{C}$, confirming that lyophilisation with glucose as cryoprotectant was not robust (data not shown). Evidently, negligible variation of lyophilisation pro- duced inappropriate products, probably due to high water residues that acted as plasticisers to the system. The formation of collapsed cakes while using glucose as cryoprotectant has already been reported in the literature. ${ }^{27)}$

Stability Studies After 7 months of storage in closed vials at $4{ }^{\circ} \mathrm{C}$, moisture content increased in all lyophilisates (Fig. 3b). The highest moisture content (up to 9\%) was observed for Lipoid S100 lecithin NPs (C1) when both cryoprotectants were used. Generally, water sorption during the 7-month storage was more pronounced for lyophilisates with glucose than for lyophilisates with trehalose. Despite the increase in moisture content, all samples preserved the cake appearance that they had immediately after the freeze-drying process. All lyophilisates could easily be redispersed and no aggregation of the particles after reconstitution was observed. For both cryoprotectants used, samples were recovered with the acceptable NP size and size distribution (Fig. 5 ). However, in some cases notable changes in mean particle size and particle size distribution could be observed, depending on the composition of the NP formulation. Thus, the initial size of lecithin NPs prepared with Lipoid S45 (sample $\mathrm{C} 1 ; 90.5 \pm 1.5 \mathrm{~nm}$ ) increased to $182.4 \pm 1.3$ and $129.2 \pm 0.5$ $\mathrm{nm}$ after 7 months of storage of lyophilisates with trehalose and glucose $(2.5 \% ; \mathrm{w} / \mathrm{v})$, respectively. These results are consistent with the results obtained when lyophilisates were redispersed immediately after freeze-drying, as the increase in particle size for the mentioned samples (up to 151.3 \pm 5.9 and 101.2 $\pm 2.1 \mathrm{~nm}$, respectively) was observed (Fig. 1). In case of lecithin/chitosan NPs (A2 and C2), no notable changes in particle diameter occurred after the 7-month storage.

As for particle size distribution, PDI values of all samples except for Lipoid S100/chitosan NPs (A2) remained below 0.40. For Lipoid S45 lecithin NPs (C1) freeze-dried in the presence of trehalose and glucose $(2.5 \%$; w/v), PDI values obtained for samples resuspended after the 7-month storage ( 0.34 and 0.33 , respectively) were notably higher than PDI values obtained when lyophilisates were redispersed immediately after freeze-drying ( 0.27 and 0.23 , respectively).

As for the zeta potential value, lecithin/chitosan NPs showed a slight decrease of the surface charge after 7 months of storage at $4{ }^{\circ} \mathrm{C}$. In the case of NPs freeze-dried with trehalose, the decrease in the surface charge could be explained by the formation of hydrogen bonds between trehalose and positively charged amino groups of chitosan, which resulted in partial neutralisation of NP surface charge. A similar reduction of zeta potential of solid lipid nanoparticles after freeze-drying in the presence of trehalose was already reported. ${ }^{27)}$ The decrease in zeta potential was attributed to the formation of hydrogen bonds between trehalose and cationic lipid head groups. In the case of NPs freeze-dried with glucose, the decrease in surface charge might be attributed to the formation of a Schiff's base through the reaction between reducing groups of glucose and amino groups of chitosan that are responsible for the NP positive surface charge. ${ }^{28)}$

Based on the results on melatonin content, we conclude that lyophilised NPs stored for 7 months at $4{ }^{\circ} \mathrm{C}$ were capable of retaining the entrapped melatonin.

Thermal Properties and X-Ray Analyses Solid-state properties of the lyophilised formulations after 7 months of storage reveal that phase separation was propagated in lechitin NPs lyophilisated with trehalose. The reverse heat flow 
(a)

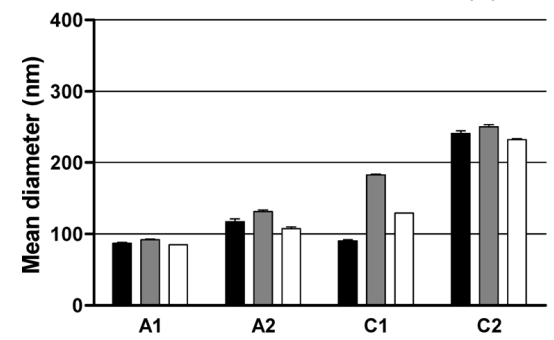

(c)

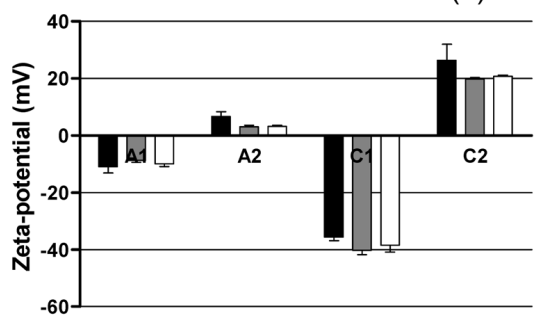

(b)

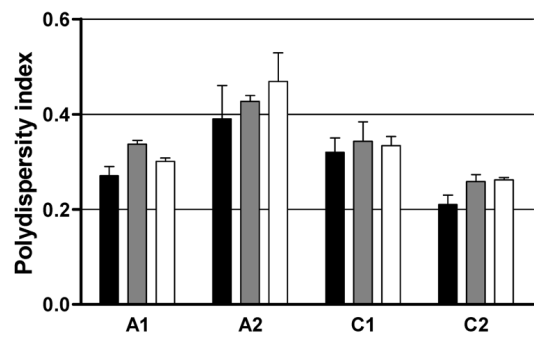

(d)

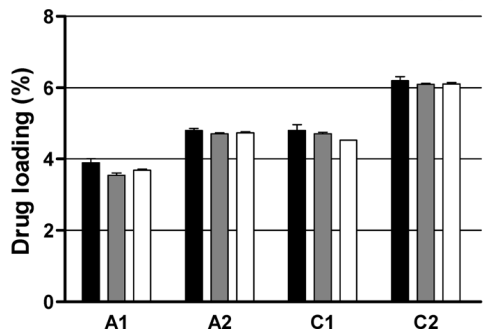

Fig. 5. Mean Diameter (a), Polydispersity (b), Zeta Potential (c) and Drug Loading (d) of Melatonin-Loaded NPs before Freeze-Drying (ם) and after Freeze-Drying in the Presence of Trehalose $(2.5 \%(\mathrm{w} / \mathrm{v})$, $\square)$ or Glucose $(2.5 \%(\mathrm{w} / \mathrm{v}) \square)$ and Rehydration in the Original Volume after Storage for 7 Months at $4{ }^{\circ} \mathrm{C}$

Data are expressed as mean \pm S.D. $(n=3)$.

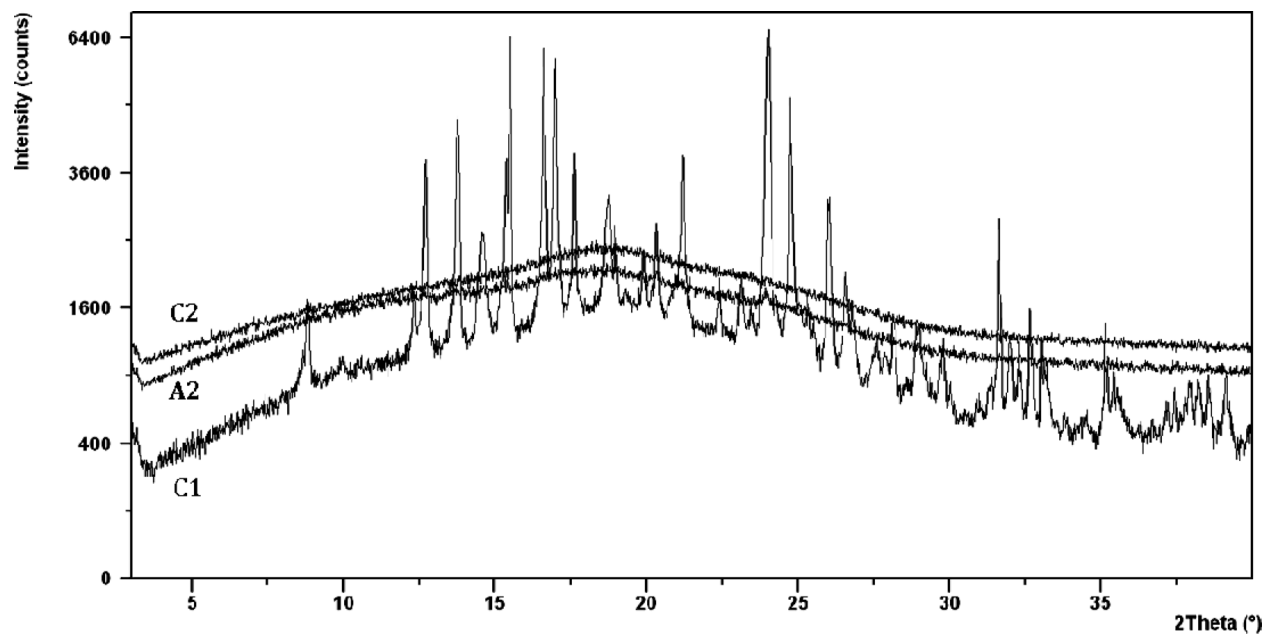

Fig. 6. X-Ray Diffractograms Obtained for the Representative Freeze-Dried NP Formulations with Trehalose after 7 Months of Storage at $4{ }^{\circ} \mathrm{C}$

curves (Fig. 4b) detected two distinct thermal events, confirming the existence of NP-enriched and trehalose-enriched phases. A thermogram of C1 NPs showed the glass transition at $32{ }^{\circ} \mathrm{C}$ that was attributed to the NP-enriched region and a sharp endothermic peak at $99{ }^{\circ} \mathrm{C}$ attributed to the hydrated trehalose. ${ }^{25,29)}$ Water residues were exceptionally high, causing formation of trehalose hydrate ${ }^{29,30}$ (as confirmed by Xray analysis provided in Fig. 6) with an additional impact on the glass transition of lecithin NPs. The thermogram of A1 NPs also showed a glass transition at $54{ }^{\circ} \mathrm{C}$ that was attributed to the NP-enriched region and a glass transition at $120^{\circ} \mathrm{C}$ being ascribed to the amorphous trehalose. This formulation also had a lower amount of residual water, so the trehalose remained intact and amorphous. The reverse heat flow curves obtained for the lyophilised lecithin/chitosan NPs with trehalose confirmed that no major change appeared upon storage. X-Ray analyses (Fig. 6) additionally substanti- ated that the representative freeze-dried NP remained amorphous upon storage.

\section{Conclusion}

In this study, a freeze-drying process of melatonin-loaded lecithin/chitosan NPs was established. A suspension of melatonin-loaded lecithin/chitosan NPs at the concentration of $0.2 \%(\mathrm{w} / \mathrm{v})$ can be successfully freeze-dried in the presence of trehalose $(2.5 \%, \mathrm{w} / \mathrm{v})$. The NP lyophilisate can be stored in solid form for 7 months while retaining NP physico-chemical properties as well as the encapsulated drug.

Acknowledgements This work was supported by Grant 006-00611171244 of the Ministry of Science, Education and Sports of the Republic of Croatia.

\section{References}

1) Sonvico F., Cagnani A., Rossi A., Motta S., Di Bari M. T., Cavatorta 
F., Alonso M. J., Deriu A., Colombo P., Int. J. Pharm., 324, 67-73 (2006).

2) Gerelli Y., Barbieri S., Di Bari M. T., Deriu A., Cantù L., Brocca P., Sonvico F., Colombo P., May R., Motta S., Langmuir, 24, $11378-$ $11384(2008)$

3) Singla A. K., Chawla M., J. Pharm. Pharmacol., 53, 1047-1067 (2001).

4) Kelmann R. G., Kuminek G., Teixeira H. F., Koester L. S., Int. J. Pharm., 342, 231-239 (2007)

5) Pavelić Z., Škalko-Basnet N., Filipović-Grčić J., Martinac A., Jalšenjak I., J. Controlled Release, 106, $34-43$ (2005).

6) Ceraulo L., Fanara S., Turco Liveri V., Ruggirello A., Panzeri W., Mele A., Colloids Surf. A Physicochem. Eng. Asp., 316, 307-312 (2008).

7) Schubert M. A., Harms M., Müller-Goymann C. C., Eur. J. Pharm. Sci., 27, 226-236 (2006).

8) Hafner A., Lovrić J., Voinovich D., Filipović-Grčić J., Int. J. Pharm., 381, 205-213 (2009).

9) Senyigit T., Sonvico F., Barbieri S., Ozer O., Santi P., Colombo P., J. Controlled Release, 142, 368-373 (2010).

10) Abdelwahed W., Degobert G., Stainmesse S., Fessi H., Adv. Drug Deliv. Rev., 58, 1688-1713 (2006).

11) Allison S. D., Molina M. C., Anchordoquy T. J., Biochim. Biophys. Acta, 1468, 127-138 (2000)

12) Molina M. C., Armstrong T. K., Zhang Y., Patel M. M., Lentz Y. K., Anchordoquy T. J., J. Pharm. Sci., 93, 2259-2273 (2004).

13) Zhang L., Liu L., Qian Y., Chen Y., Eur. J. Pharm. Biopharm., 69, $750-759$ (2008)

14) Sameti M., Bohr G., Ravi Kumar M. N., Kneuer C., Bakowsky U., Nacken M., Schmidt H., Lehr C. M., Int. J. Pharm., 266, 51-60 (2003).
15) Schaffazick S. R., Pohlmann A. R., de Cordova C. A., CreczynskiPasa T. B., Guterres S. S., Int. J. Pharm., 289, 209-213 (2005).

16) Crowe L. M., Reid D. S., Crowe J. H., Biophys. J., 71, 2087-2093 (1996).

17) Yu L., Adv. Drug Deliv. Rev., 48, 27-42 (2001).

18) Akagi T., Kaneko T., Kida T., Akashi M., J. Controlled Release, 108, $226-236(2005)$.

19) Prego C., Paolicelli P., Díaz B., Vicente S., Sánchez A., GonzálezFernández A., Alonso M. J., Vaccine, 28, 2607-2614 (2010).

20) Sonaje K., Chen Y. J., Chen H. L., Wey S. P., Juang J. H., Nguyen H. N., Hsu C. W., Lin K. J., Sung H. W., Biomaterials, 31, 3384-3394 (2010).

21) Konan Y. N., Gurny R., Allémann E., Int. J. Pharm., 233, 239-252 (2002).

22) Abdelwahed W., Degobert G., Fessi H., Eur. J. Pharm. Biopharm., 63 , 87-94 (2006).

23) Holzer M., Vogel V., Mäntele W., Schwartz D., Haase W., Langer K., Eur. J. Pharm. Biopharm., 72, 428-437 (2009).

24) Di Tommaso C., Como C., Gurny R., Möller M., Eur. J. Pharm. Sci., 40, 38-47 (2010).

25) Taylor L. S., York P., J. Pharm. Sci., 87, 347-355 (1998).

26) Adler M., Lee G., J. Pharm. Sci., 88, 199-208 (1999).

27) del Pozo-Rodríguez A., Solinís M. A., Gascón A. R., Pedraz J. L., Eur. J. Pharm. Biopharm., 71, 181-189 (2009).

28) Hinrichs W. L., Sanders N. N., De Smedt S. C., Demeester J., Frijlink H. W., J. Controlled Release, 103, 465-479 (2005).

29) Surana R., Pyne A., Suryanarayanan R., Pharm. Res., 21, 1167-1176 (2004).

30) Chen C., Han D., Cai C., Tang X., J. Controlled Release, 142, 299311 (2010). 